RUNHETC-00-45

hep-th/0011125

\title{
*-Trek II:
}

$*_{n}$ Operations, Open Wilson Lines and the Seiberg-Witten Map

\author{
Hong Liu* \\ New High Energy Theory Center \\ Rutgers University \\ 126 Frelinghuysen Road \\ Piscataway, NJ $08854 \quad$ USA
}

\begin{abstract}
Generalizations of the $*$-product (e.g. $n$-ary $*_{n}$ operations) appear in various places in the discussion of noncommutative gauge theories. These include the one-loop effective action of noncommutative gauge theories, the couplings between massless closed and open string modes, and the Seiberg-Witten map between the ordinary and noncommutative Yang-Mills fields. We propose that the natural way to understand the $*_{n}$ operations is through the expansion of an open Wilson line. We establish the connection between an open Wilson line and the $*_{n}$ operations and use it to (I) write down a gauge invariant effective action for the one-loop $F^{4}$ terms in the noncommutative $\mathcal{N}=4 \mathrm{SYM}$ theory; (II) find the gauge invariant couplings between the noncommutative SYM modes and the massless closed string modes in flat space; (III) propose a closed form for the Seiberg-Witten map in the $U(1)$ case.
\end{abstract}

\footnotetext{
*liu@physics.rutgers.edu
} 


\section{Contents}

$\begin{array}{ll}\text { 1. Introduction } & 1\end{array}$

2. Open Wilson Lines and the $*_{n}$ Operations 5

3. One-loop $\hat{F}^{4}$ Terms in $\mathcal{N}=4$ Noncommutative SYM 9

3.1 Gauge Invariant Completion of the One-loop $\hat{F}^{4}$ Terms 9

3.2 Closed String Couplings to the Noncommutative Yang-Mills Modes 11

4. The Seiberg-Witten Map 13

4.1 The Seiberg-Witten Map to First Order in $\theta \quad 13$

4.2 A Proposal for the Seiberg-Witten Map 15

5. Equivalence of Born-Infeld Actions Revisited 18

$\begin{array}{lr}\text { 6. Discussion } & 20\end{array}$

A. Notations and Conventions 22

B. The $*_{n}$ Operations $\quad 23$

C. An Alternative Derivation of the Seiberg-Witten Map in the Slowlyvarying Field Case $\quad 25$

\section{Introduction}

Noncommutative gauge theories have attracted much interest recently. They appear naturally in various decoupling limits of the worldvolume theories of D-branes in a background NS-NS $B$-field $[1,2,3,4,5,6]$ and provide simplified settings for studying nonlocal effects in string theory.

In noncommutative gauge theories, gauge invariance becomes subtle. When there is only adjoint matter, translations along noncommutative directions are a subset of gauge transformations, and thus there are no gauge invariant local operators in position 
space $[7,8] .{ }^{1}$ It turns out that noncommutative gauge theories allow a new type of gauge invariant objects which are localized in momentum space. They are open Wilson lines [11]. An open Wilson line is gauge invariant [11, 12, 8, 13] provided that the distance between the end points of the line $\Delta x$ and its momentum $k$ satisfy the relation

$$
\Delta x^{\mu}=\theta^{\mu \nu} k_{\nu}
$$

where $\theta$ is the noncommutative parameter of the theory (see Appendix A for our notations). Attaching local operators which transform adjointly under the gauge transformations to an open Wilson line also yields gauge invariant operators [13, 14].

Recently, we have computed the one-loop four gauge boson scattering amplitude in noncommutative $\mathcal{N}=4 \mathrm{SYM}$ theory and extracted the corresponding contribution to the one-loop effective action in a momentum expansion $[15]^{2}$. It was found that the one-loop low energy effective action involves generalizations of $*$-products. To each non-planar diagram, depending on the number $n$ of external vertex operator insertions on each boundary, there is a corresponding $*_{n} n$-ary operation ${ }^{3}$. In particular a careful analysis of the one-loop $F^{4}$ terms found that the resulting effective action does not respect the gauge symmetry of the tree-level action when expressed in terms of $*_{2}$ and $*_{3}$ operations. For explicit formulas for the $*_{2}$ and $*_{3}$ operations and the definition of general $*_{n}$ operations, see Appendix B.

The $*_{2}, *_{3}$ operations were also found in the tree-level closed-open amplitudes in the presence of a $B$-field $[17,19]$ and in the Seiberg-Witten map [6] between the ordinary and noncommutative gauge field variables $[17,20] . *_{n}$ operations should also be present in the tree-level amplitudes between one closed and $n$ open string modes in the presence of a $B$-field.

In this paper we propose that a natural way to understand the family of $*_{n}$ operations is through the open Wilson lines ${ }^{4}$ and study some implications. In particular, we introduce a family of gauge invariant operators by integrating the insertion points of the attached operators along the path of a straight open Wilson line. As an example, if $\mathcal{O}_{i}, i=1, \cdots, n$ are local operators which transform adjointly under the gauge

\footnotetext{
${ }^{1}$ Matter in other representations can sometimes be used to construct gauge invariant local operators. $[9,10]$

${ }^{2}$ The calculation in [15] was performed in string theory and then reduced to field theory result by taking the $\alpha^{\prime} \rightarrow 0$ limit. The computations in field theory were later performed in [16] using superspace techniques.

$3 *_{2}$, also called $*^{\prime}$ in $[17,15]$ was first found in [17]. It also appeared in [18].

${ }^{4}$ Similar ideas were also explored recently in $[20]$.
} 
transformation, we define

$$
\begin{aligned}
\mathcal{Q}(k) & =\int d^{4} x\left(\prod_{i=1}^{n} \int_{0}^{1} d \tau_{i}\right) P_{*}\left[W(x, C) \prod_{i=1}^{n} \mathcal{O}_{i}\left(x+\xi\left(\tau_{i}\right)\right)\right] * e^{i k \cdot x} \\
& \equiv \int d^{4} x L_{*}\left[W(x, C) \prod_{i=1}^{n} \mathcal{O}_{i}(x)\right] * e^{i k \cdot x}
\end{aligned}
$$

where $W(x, C)$ is a straight open Wilson line [13] with its path $C$ parameterized by $\xi(\tau), 0 \leq \tau \leq 1$ and $P_{*}$ denotes path ordering with respect to the $*$-product. In the second line we have introduced a short-hand notation $L_{*}$ to denote the integrations together with the path ordering procedure. To the lowest order in the expansion of the Wilson line in terms of $\hat{A}$, it can be shown that

$$
\mathcal{Q}(k)=\int d^{4} x *_{n}\left[\mathcal{O}_{1}(x), \cdots, \mathcal{O}_{n}(x)\right] e^{i k \cdot x}+O(\hat{A})
$$

From the relation between (1.2) and (1.3) we can write down a gauge invariant completion of the $F^{4}$ terms in $[15,16]$ using operators of type (1.2), the expansion of which in $\hat{A}$ reduces to the results of $[15,16]$ at the lowest order. Higher order terms in the expansion can be explicitly checked by looking at the one-loop amplitudes of higher number of external vectors and will be discussed in a separate place [21].

Since the $F^{4}$ term in $\mathcal{N}=4$ noncommutative SYM theory also has the interpretation in string theory as a tree-level process for exchanging the massless closed string modes between sources on distant branes, the completion helps to identify the gauge invariant operators to which the closed string modes couple. For example, the coupling between the dilaton $\phi$ and the modes of the noncommutative $\mathcal{N}=4 \mathrm{SYM}$ is:

$$
S_{I}=\frac{1}{\left(2 \pi G_{s}\right)} \int d^{4} k \sqrt{\operatorname{det} G} \phi(-k) \mathcal{O}_{\phi}(k)
$$

with

$$
\begin{aligned}
\mathcal{O}_{\phi}(k) & =\frac{1}{4} \operatorname{Tr} \int d^{4} x L_{*}\left[W(x, C) \hat{F}_{\mu \nu}(x) \hat{F}^{\mu \nu}(x)\right] * e^{i k \cdot x} \\
& =\frac{1}{4} \operatorname{Tr} \int d^{4} x\left[\hat{F}_{\mu \nu}(x) *_{2} \hat{F}^{\mu \nu}(x)+\theta^{\lambda \rho} \partial_{\rho}\left(*_{3}\left[\hat{F}_{\mu \nu}(x), \hat{F}^{\mu \nu}(x), \hat{A}_{\lambda}(x)\right]\right)+\cdots\right] * e^{i k \cdot x}
\end{aligned}
$$

where $G_{s}$ and $G_{\mu \nu}$ are the open string coupling and metric, $\hat{F}^{\prime}$ 's are contracted by the open string metric, and we have used the $L_{*}$ notation defined in (1.2). In (1.5) each $\hat{F}$ is integrated separately along the line. The resulting operator is different from the one 
obtained by binding them together [13]. The coupling (1.4) is written in the momentum space and there appears no simple counterpart of it in the position space ${ }^{5}$. The above identification should be useful for understanding the operator-field correspondence in the noncommutative version of the AdS/CFT correspondence [22, 23].

It is tempting to speculate that the $*_{n}$ operations appearing in the Seiberg-Witten map may also be attributed to the open Wilson lines. At a heuristic level, this expectation is supported by the following reasoning. In noncommutative field theories the elementary quanta are no longer point particles; instead they are one dimensional extended objects (with no oscillations) with the property that their extension is proportional to the center of mass momentum, i.e. given by a relation $[24,25]$,

$$
\Delta x^{\mu}=\theta^{\mu \nu} k_{\nu}
$$

Intuitively this can be understood as an electric dipole moving in a background magnetic field at its lowest Landau level [24, 26, 27]. The coincidence of (1.6) with the gauge invariance condition (1.1) of an open Wilson line suggest that we may imagine an elementary quantum macroscopically as an open Wilson line of the same momentum. The field strength $F_{\mu \nu}(k)$ of momentum $k$ for an ordinary $U(1)$ gauge field is invariant under noncommutative gauge transformations and creates an elementary quantum of momentum $k$. It seems physically appealing that $F^{\mu \nu}(k)$ be expressible in terms of an open Wilson line of length (1.6) with possible operator insertions.

We shall argue that this is indeed the case based on the earlier results on the Seiberg-Witten map $[17,28,29,30,31,32,20]$ and the connection between the $*_{n}$ operations and the open Wilson lines. We propose a closed form for the expression of an ordinary $U(1)$ field strength in terms of the noncommutative Yang-Mills field,

$$
F_{\mu \nu}(k)=\int d^{4} x L_{*}\left[\sqrt{\operatorname{det}(1-\theta \hat{F})}\left(\frac{1}{1-\hat{F} \theta} \hat{F}\right)_{\mu \nu}(x) W(x, C)\right] e^{i k \cdot x}
$$

In (1.7), $W(x, C)$ is a straight open Wilson line, the determinant and rational function of $\hat{F}$ should be understood as a power series expansion, and $L_{*}$ acts on each term in the expansion as defined in (1.2). (1.7) is gauge invariant by construction and recovers the results of $[17,20]$ upon expanding to cubic order in $\hat{A}$. (1.7) also has the correct small $\theta$ limit, in which case the $*$-product can be replaced by the Poisson Brackets. However, we are unable to prove it to all orders in this paper.

\footnotetext{
${ }^{5}$ That the couplings between noncommutative Yang-Mills and closed string modes ought to be in momentum space rather than in coordinate space was pointed out in $[8,13]$.
} 
In the situation where we can ignore the derivatives of the field strength, equation (1.7) simplifies and can be written as:

$$
F_{\mu \nu}(X(x))=\left(\frac{1}{1-\hat{F} \theta} \hat{F}\right)_{\mu \nu}(x)
$$

where

$$
X^{\mu}(x)=x^{\mu}+\theta^{\mu \nu} \hat{A}_{\nu}(x)
$$

When applied to the Born-Infeld action, (1.8) gives a direct and simple derivation of the equivalence between the noncommutative and ordinary Born-Infeld actions [6].

The plan of the paper is as follows. In section 2, we point out the close relationship between the $*_{n}$ operations and a straight open Wilson line. In section 3 , we propose a gauge invariant $F^{4}$ effective action for $\mathcal{N}=4$ noncommutative SYM theory and from it work out the couplings between massless closed string modes and the noncommutative Yang-Mills modes. In section 4 we motivate a closed form for the Seiberg-Witten map in the $U(1)$ case. In section 5 we derive the Seiberg-Witten map in the case of a slowly varying field strength and revisit the equivalence between the ordinary and noncommutative Born-Infeld actions. In particular we clarify certain aspects of the equivalence. We close with some remarks in section 6. We have included a number of appendices. In Appendix A we introduce our conventions for the $*$-product and noncommutative gauge theory. In Appendix B we review the definition for the $*_{n}$ operations and give some explicit expressions for $n=2,3$. Appendix $\mathrm{C}$ contains an alternative derivation of the Seiberg-Witten map in the slowly-varying field case.

\section{Open Wilson Lines and the $*_{n}$ Operations}

In this section we derive some results about the open Wilson lines and the $*_{n}$ operations. Similar ideas were also explored in [20]. Our conventions for the *-product and noncommutative gauge theories are given in Appendix A. For a review of the $*_{n}$ operations refer to Appendix B. Throughout the paper we will use $k_{1} \times k_{2} \equiv k_{1 \mu} \theta^{\mu \nu} k_{2 \nu}$.

A straight open Wilson line of momentum $k$ is given by

$$
W_{k}(C)=\operatorname{Tr} \int d^{4} x W(x, C) * e^{i k \cdot x}
$$

where

$$
W(x, C)=P_{*} \exp \left(i \int_{0}^{1} d \sigma \partial_{\sigma} \xi^{\mu}(\sigma) \hat{A}_{\mu}(x+\xi(\sigma))\right)
$$


and the path $C$ is a straight line

$$
\xi^{\mu}(\sigma)=\theta^{\mu \nu} k_{\nu} \sigma
$$

In the above $P_{*}$ denotes the path ordering with respect to the $*$-product. Under a gauge transformation the Wilson line (2.2) transforms as $\left(l^{\mu}=\theta^{\mu \nu} k_{\nu}\right)$

$$
W(x, C) \longrightarrow U(x) * W(x, C) * U(x+l)^{\dagger}
$$

and the "momentum-space" representation (2.1) is gauge invariant $[11,12,8,13]$. Similarly for any local operator $\mathcal{O}$ which transforms adjointly under the gauge transformation, the following operator is gauge invariant [13]

$$
\mathcal{O}(k)=\operatorname{Tr} \int d^{4} x P_{*}[W(x, C) \mathcal{O}(y)] * e^{i k \cdot x},
$$

where $y$ is some point on the path (2.3) of the Wilson line.

We now introduce gauge invariant operators of the type

$$
\begin{aligned}
\mathcal{Q}(k) & =\int d^{4} x\left(\prod_{i=1}^{n} \int_{0}^{1} d \tau_{i}\right) P_{*}\left[W(x, C) \prod_{i=1}^{n} \mathcal{O}_{i}\left(x+\xi\left(\tau_{i}\right)\right)\right] * e^{i k \cdot x} \\
& \equiv \int d^{4} x L_{*}\left[W(x, C) \prod_{i=1}^{n} \mathcal{O}_{i}(x)\right] * e^{i k \cdot x}
\end{aligned}
$$

where $\mathcal{O}_{i}$ transform adjointly under the gauge transformation and $\xi(\sigma)$ is given by (2.3). In (2.5) we integrated the insertion points of the external operators along the path and this may be interpreted as a most "democratic" way of attaching external operators to an open Wilson line. When there is only a single operator (i.e. $n=1$ in $(2.5)$ ), the integration can be dropped since insertion at any point of the straight line is equivalent to another [13]. Similarly for $n>1$, we can choose an arbitrary operator and fix its position at e.g. $\tau=0$.

Equation (2.5) can be expanded in terms of a power series in $\hat{A}$,

$$
Q(k)=Q_{0}(k)+Q_{1}(k, \hat{A})+\cdots
$$

where $Q_{n}(k)$ involves $n$ powers of $\hat{A}$. For example, $Q_{0}(k)$ is given by

$$
Q_{0}(k)=\operatorname{Tr} \int d^{4} x\left(\prod_{i=1}^{n} \int_{0}^{1} d \tau_{i}\right) P_{*}\left[\prod_{i=1}^{n} \mathcal{O}_{i}\left(x+\xi\left(\tau_{i}\right)\right)\right] * e^{i k \cdot x}
$$


Let us now look at the structure of each term in (2.6) more explicitly. We shall first look at the simpler $U(1)$ case. Fourier-transforming $\mathcal{O}_{i}$ in (2.7)

$$
\begin{aligned}
\mathcal{O}_{i}\left(x+\xi\left(\tau_{i}\right)\right) & =\int \frac{d^{4} k_{i}}{(2 \pi)^{4}} \mathcal{O}_{i}\left(k_{i}\right) e^{-i k_{i} \cdot(x+\xi(\tau))} \\
& =\int \frac{d^{4} k_{i}}{(2 \pi)^{4}} \mathcal{O}_{i}\left(k_{i}\right) e^{-i k_{i} \cdot x-i\left(k_{i} \times k\right) \tau_{i}}
\end{aligned}
$$

and integrating over $x$, we get

$$
Q_{0}(k)=\left(\prod_{i=1}^{n} \int \frac{d^{4} k_{i}}{(2 \pi)^{4}}\right)(2 \pi)^{4} \delta^{(4)}\left(k-\sum_{i=1}^{n} k_{i}\right) \mathcal{O}_{1}\left(k_{1}\right) \cdots \mathcal{O}_{n}\left(k_{n}\right) J_{n}\left(k_{1}, \cdots, k_{n}\right)
$$

where $J_{n}$ is given by $\left(\tau_{i j}=\tau_{i}-\tau_{j}\right)$ :

$$
J_{n}\left(k_{1}, \cdots, k_{n}\right)=\int_{0}^{1} d \tau_{1} \cdots \int_{0}^{1} d \tau_{n} \exp \left[-\frac{i}{2} \sum_{i<j}^{n}\left(k_{i} \times k_{j}\right)\left(2 \tau_{i j}-\epsilon\left(\tau_{i j}\right)\right)\right]
$$

In reaching (2.10) from (2.7) we have used the momentum conservation condition $k=$ $\sum_{i=1}^{n} k_{i}$ and the identity

$$
\sum_{i=1}^{n}\left(k_{i} \times k\right) \tau_{i}=\sum_{i<j}^{n}\left(k_{i} \times k_{j}\right)\left(\tau_{i}-\tau_{j}\right) .
$$

Equation (2.11) is precisely the momentum space kernel of the $*_{n}$ operation on the space of $n$-functions defined in [15] (see Appendix B). In coordinate space $Q_{0}(2.10)$ can be written in the form

$$
Q_{0}(k)=\int d^{4} x Q_{0}(x) e^{i k \cdot x}, \quad Q_{0}(x)=*_{n}\left[\mathcal{O}_{1}(x), \cdots, \mathcal{O}_{n}(x)\right]
$$

Using the same manipulations, we find the $m$ th order term in (2.6) can be rewritten as

$$
Q_{m}(k)=\frac{i^{m}}{m !}\left(\prod_{i=1}^{n+m} \int \frac{d^{4} k_{i}}{(2 \pi)^{4}}\right) \mathcal{O}_{1}\left(k_{1}\right) \cdots \mathcal{O}_{n}\left(k_{n}\right) M\left(k_{n+1}\right) \cdots M\left(k_{n+m}\right) J_{n+m}\left(k_{1}, \cdots, k_{m+n}\right)
$$

where

$$
M\left(k_{i}\right)=\theta^{\mu \nu} k_{\mu} \hat{A}_{\nu}\left(k_{i}\right)
$$


and we have omitted the $(2 \pi)^{4} \delta^{(4)}\left(k-\sum_{i=1}^{n+m} k_{i}\right)$ factor inside the integral to make the formula compact. In coordinate space, equation (2.14) can be written as

$$
Q_{m}(x)=\frac{1}{m !}(\theta \partial)^{\mu_{1}} \cdots(\theta \partial)^{\mu_{m}} *_{m+n}\left[\mathcal{O}_{1}(x), \cdots, \mathcal{O}_{n}(x), \hat{A}_{\mu_{1}}(x), \cdots, \hat{A}_{\mu_{m}}(x)\right]
$$

with $(\theta \partial)^{\mu}=\theta^{\mu \nu} \partial_{\nu}$.

The explicitly expression for $J_{n}$ and the corresponding $*_{n}$ operation in the $U(1)$ case can be found straightforwardly from (2.11). However, when $n$ becomes large the structure becomes quite complicated and hard to extract useful information. Here we list some useful properties:

1. $*_{n}$ is fully symmetric, i.e.

$$
*_{n}(\cdots, f, \cdots, g, \cdots)=*_{n}(\cdots, g, \cdots, f, \cdots)
$$

This property also applies to the general $U(N)$ case.

2. $J_{n}$ satisfies a descent relation,

$$
J_{n}\left(k_{1}, \cdots, k_{n}\right)=\frac{2}{k_{n} \times k} \sum_{j=1}^{n-1} \sin \frac{k_{n} \times k_{j}}{2} J_{n-1}\left(k_{1}, \cdots, k_{j}+k_{n}, \cdots, k_{n-1}\right)
$$

with $k=\sum_{i=1}^{n} k_{i}$. In position space this implies ${ }^{6}$

$$
\theta^{i j} \partial_{j}\left[*_{n}\left(f_{1}(x), \cdots, f_{n}(x), \partial_{i} g(x)\right)\right]=i \sum_{j=1}^{n-1} *_{n-1}\left(f_{1}(x), \cdots,\left[f_{j}, g\right], \cdots, f_{n-1}(x)\right)
$$

where

$$
[f, g]=f(x) * g(x)-g(x) * f(x)
$$

The explicit expressions for $J_{2}, *_{2}$ and $J_{3}, *_{3}$ are listed in the Appendix B.

More generally for a $U(N)$ noncommutative gauge theory, the structures of $J_{n}$ and $*_{n}$ are more complicated, since we have to include the ordering of matrices in $(2.11)$ and (2.14). For example, let $\mathcal{O}_{i}=\mathcal{O}_{i}^{a_{i}} T^{a_{i}}$ where $T^{a_{i}}$ are a set of basis for $N \times N$ Hermitian matrices, then equation (2.11) now becomes

$$
\begin{aligned}
& J_{n}\left(a_{1}, k_{1} ; \cdots ; a_{n}, k_{n}\right) \\
& =\left(\prod_{i=1}^{n} \int_{0}^{1} d \tau_{i}\right) P_{\tau}\left(T^{a_{1}} \cdots T^{a_{n}}\right) \exp \left[-\frac{i}{2} \sum_{i<j}^{n}\left(k_{i} \times k_{j}\right)\left(2 \tau_{i j}-\epsilon\left(\tau_{i j}\right)\right)\right]
\end{aligned}
$$

\footnotetext{
${ }^{6}$ The equations for $n=2,3$ were also found in [20].
} 
where $P_{\tau}$ denotes an ordering of matrices according to the ordering of $\tau_{i}$. With the above matrix ordering in mind, the formulas (2.13) and (2.16) apply to general $U(N)$ case without change.

Note in $Q_{m}, m=0,1, \cdots$ all the entries (including external operators and $\hat{A}$ 's from the Wilson line) are completely symmetric under change of orderings, as a result of $*_{n}$ operation. Therefore, (2.5) may be considered as a "generalized symmetrized trace" prescription for the algebra of the Moyal product.

\section{One-loop $\hat{F}^{4}$ Terms in $\mathcal{N}=4$ Noncommutative SYM}

\subsection{Gauge Invariant Completion of the One-loop $\hat{F}^{4}$ Terms}

In $[15,16]$, it was found that the non-planar part of one-loop $F^{4}$ terms in $\mathcal{N}=4$ noncommutative Super-Yang-Mills theory involve $*_{2}$ and $*_{3}$ operations. It was also found that if we naïvely extend the gauge invariant on-shell amplitudes off-shell using the $*_{2}$ and $*_{3}$ operations, the resulting effective action does not respect the gauge symmetry of the tree-level action. It was speculated there that it might be possible to write down a gauge invariant effective action using open Wilson lines as they are the natural gauge invariant objects of the theory. From the connections between an open Wilson line and the $*_{n}$ operations found in section 2 , we can now immediately write down such a candidate.

Let us first recall the results of [15] (see also [16]). Consider a $U(N)$ noncommutative $\mathcal{N}=4 \mathrm{SYM}$ theory which is broken by a Higgs mechanism to $U\left(N_{1}\right) \times U\left(N_{2}\right)$ $\left(N=N_{1}+N_{2}\right)$. The one-loop scattering amplitudes between massless vector bosons in the unbroken subgroups $U\left(N_{1}\right)$ and $U\left(N_{2}\right)$ are given by non-planar diagrams with intermediate loop particles massive $W$-bosons (and their super-partners). With four external vectors the amplitudes at the lowest order in momentum expansion (as compared to the mass $m$ of the $W$-bosons) give rise to the $F^{4}$ terms in the low energy effective action. The non-planar part of $F^{4}$ terms found in [15] is:

$$
\begin{aligned}
& \Gamma_{\text {1-loop }}=-\frac{1}{4 !(4 \pi)^{2}} \int d^{4} x \sqrt{\operatorname{det} G} t^{\mu \nu \rho \sigma \lambda \tau \alpha \beta} \\
& \quad \times\left\{3 \operatorname{Tr}_{U\left(N_{1}\right)}\left[F_{\mu \nu}(x) *_{2} F_{\rho \sigma}(x)\right] I_{2}\left(m, \sqrt{\overleftarrow{\partial_{\mu}}(\Theta G \Theta)^{\mu \nu} \overrightarrow{\partial_{\nu}}}\right) \operatorname{Tr}_{U\left(N_{2}\right)}\left[F_{\lambda \tau}(x) *_{2} F_{\alpha \beta}(x)\right]\right. \\
& \left.\quad-4 \operatorname{Tr}_{U\left(N_{1}\right)}\left(*_{3}\left[F_{\mu \nu}(x), F_{\rho \sigma}(x), F_{\lambda \tau}(x)\right]\right) I_{2}\left(m, \sqrt{-\partial_{\mu}(\Theta G \Theta)^{\mu \nu} \partial_{\nu}}\right) \operatorname{Tr}_{U\left(N_{2}\right)}\left(F_{\alpha \beta}(x)\right)\right\} \\
& +\left(N_{1} \rightarrow N_{2}\right)
\end{aligned}
$$


where the traces are taken in the fundamental of the indicated subgroup and

$$
I_{2}(m, x) \equiv\left(\frac{x}{2 m}\right)^{2} K_{2}(m x)
$$

with $K_{2}$ a modified Bessel function. In (3.1a) $t^{\mu \nu \rho \sigma \lambda \tau \alpha \beta}$ is a tensor composed of open string metrics and when contracted with four anti-symmetric tensors gives the structure

$$
\begin{aligned}
& t^{\mu \nu \rho \sigma \lambda \tau \alpha \beta} M_{1 \mu \nu} M_{2 \rho \sigma} M_{3 \lambda \tau} M_{4 \alpha \beta} \\
& =2^{4}\left(M_{1 \mu}{ }^{\lambda} M_{2 \lambda}{ }^{\alpha} M_{3 \alpha}{ }^{\nu} M_{4 \nu}{ }^{\mu}-\frac{1}{4} M_{1 \mu \nu} M_{2}^{\nu \mu} M_{3 \tau \sigma} M_{4}^{\sigma \tau}+2 \text { permutations }\right) .
\end{aligned}
$$

where the two permutations are given by changing the ordering from (1234) to (1342) and (1423).

To write down a gauge invariant version of (3.1), we define

$$
\begin{aligned}
\mathcal{O}_{\mu \nu \rho \sigma}(k) & =\int d^{4} x L_{*}\left[W(x, C) \hat{F}_{\mu \nu}(x) \hat{F}_{\rho \sigma}(x)\right] * e^{i k \cdot x} \\
\mathcal{O}_{\mu \nu \rho \sigma \lambda \tau}(k) & =\int d^{4} x L_{*}\left[W(x, C) \hat{F}_{\mu \nu}(x) \hat{F}_{\rho \sigma}(x) \hat{F}_{\lambda \tau}(x)\right] * e^{i k \cdot x},
\end{aligned}
$$

where according to (1.2) each $\hat{F}$ is integrated separately along the line ${ }^{7}$. From equation (2.10) and (2.13), the $\hat{F}^{4}$ effective action (3.1) can be recovered from the lowest order term of the following gauge invariant action

$$
\begin{aligned}
\Gamma_{1-\text { loop }} & =-\frac{1}{4 !(4 \pi)^{2}} \int \frac{d^{4} k}{(2 \pi)^{4}} t^{\mu \nu \rho \sigma \lambda \tau \alpha \beta}\left[3 \operatorname{Tr}_{U\left(N_{1}\right)} \mathcal{O}_{\mu \nu \rho \sigma}(k) I_{2}(m,|\Delta x|) \operatorname{Tr}_{U\left(N_{2}\right)} \mathcal{O}_{\lambda \tau \alpha \beta}(-k)\right. \\
& \left.-4 \operatorname{Tr}_{U\left(N_{1}\right)} \mathcal{O}_{\mu \nu \rho \sigma \lambda \tau}(k) I_{2}(m,|\Delta x|) \operatorname{Tr}_{U\left(N_{2}\right)} \hat{F}_{\alpha \beta}(-k)\right]+\left(N_{1} \leftrightarrow N_{2}\right)
\end{aligned}
$$

where $|\Delta x|=\left|\Theta_{\mu \nu} k_{\nu}\right|$ and $I_{2}(m,|\Delta x|)$ is given by (3.1b).

In the above we have written the gauge invariant effective action in momentum space as the gauge invariant operators (3.3) are well-defined local operators in momentum space. They do not appear to have sensible Fourier transformations to position space. This is a reflection of the fact that translations are part of the gauge symmetry $[8,7]$. The higher order terms in $(3.5)$ can be checked explicitly by computing the higher-point amplitudes and will be discussed elsewhere [21].

\footnotetext{
${ }^{7}$ Note in $\mathcal{N}=4$ noncommutative SYM theory, the open Wilson lines in (3.3) might also contain scalar fields $\Phi$ or fermions in addition to $(2.2)$, e.g. [33, 34, 8]

$$
W(x, C)=P_{*} \exp \left[i \int_{0}^{1} d \sigma\left(\partial_{\sigma} \xi^{\mu}(\sigma) \hat{A}_{\mu}(x+\xi(\sigma))+\left|\partial_{\sigma} \xi\right| \Omega \cdot \Phi(x+\xi(\sigma))\right)\right]
$$

with $\Omega$ a unit vector on $S^{5}$.
} 


\subsection{Closed String Couplings to the Noncommutative Yang-Mills Modes}

The effective action (3.1), (3.5) can also be understood as a tree-level process for exchanging closed string modes between gauge invariant sources on distant branes ${ }^{8}$. It is well known that the contributions from massive modes to $F^{4}$ terms cancel among themselves [38] and only the massless modes are exchanged. From this point of view, $I_{2}(m,|\Delta x|)$ is nothing but the propagator for a masless scalar field in the transverse dimensions. The intermediate closed string modes have momentum $k_{\mu}$ along the brane directions, which appears as an effective mass term for the transverse propagator,

$$
\begin{gathered}
G(k, r)=\frac{1}{\sqrt{\operatorname{det} g}} \int \frac{d^{\tilde{d}} q}{(2 \pi)^{\tilde{d}}} \frac{e^{i q \cdot r}}{q^{2}+M^{2}} . \\
M^{2}=-k_{\mu} g^{\mu \nu} k_{\nu}=-k_{\mu} G^{\mu \nu} k_{\nu}+\left(\frac{1}{2 \pi \alpha^{\prime}}\right)^{2} k_{\mu}(\theta G \theta)^{\mu \nu} k_{\nu} \sim\left(\frac{\Delta x}{2 \pi \alpha^{\prime}}\right)^{2} .
\end{gathered}
$$

In (3.6), $\tilde{d}$ is the dimension of the transverse space, the distance between the two stacks of D-barnes is $r=2 \pi \alpha^{\prime} m$ and we have used the relation between the closed $\left(g_{\mu \nu}\right)$ and open string metrics $\left(G_{\mu \nu}\right)[6]$,

$$
g^{\mu \nu}=G^{\mu \nu}-\left(\frac{1}{2 \pi \alpha^{\prime}}\right)^{2}(\theta G \theta)^{\mu \nu}
$$

Note that we are considering the field theory limit in which $m$ and $G, \theta$ are finite while $\alpha^{\prime}, r \rightarrow 0$. It is easy to check from (3.6) and (3.1b) that

$$
I_{2}(m,|\Delta x|)=\sqrt{\operatorname{det} g} 2 \pi^{3}\left(2 \pi \alpha^{\prime}\right)^{4} G(k, r) .
$$

Using the explicit tensor structure of $t$ in (3.2), the effective action (3.5) can be rewritten as

$$
\begin{aligned}
\Gamma_{1-\text { loop }}=-2 \kappa_{10}^{2} \operatorname{det} G \frac{1}{\left(2 \pi G_{s}\right)^{2}} \int d^{4} k G(k, r)\left[\mathcal{O}_{\phi}^{(1)}(-k) \mathcal{O}_{\phi}^{(2)}(k)+\mathcal{O}_{\chi}^{(1)}(-k) \mathcal{O}_{\chi}^{(2)}(k)\right. \\
\left.+\frac{1}{2} T_{\nu}^{(1) \mu}(-k) T_{\mu}^{(2) \nu}(k)+\left.\frac{1}{2} \Sigma_{\nu}^{(1) \mu}(-k) \Sigma_{\mu}^{(2) \nu}(k)\right|_{4}\right]
\end{aligned}
$$

where

$$
2 \kappa_{10}^{2}=(2 \pi)^{7} g_{s}^{2} \alpha^{\prime 4}
$$

\footnotetext{
${ }^{8}$ For discussions in ordinary flat space or in the context of AdS/CFT correspondence, see e.g. [35, $36,37]$.
} 
and $g_{s}$ and $G_{s}$ are closed and open string couplings, which are related by [6]

$$
G_{s}=g_{s}\left(\frac{\operatorname{det} G}{\operatorname{det} g}\right)^{\frac{1}{4}}
$$

The various gauge invariant operators in (3.10) are defined by

$$
\begin{aligned}
\mathcal{O}_{\phi}(k) & =\frac{1}{4} \operatorname{Tr} \int d^{4} x L_{*}\left[W(x, C) \hat{F}_{\mu \nu}(x) \hat{F}^{\mu \nu}(x)\right] * e^{i k \cdot x} \\
\mathcal{O}_{\chi}(k) & =\frac{1}{8} \epsilon_{\mu \nu \lambda \rho} \operatorname{Tr} \int d^{4} x L_{*}\left[W(x, C) \hat{F}^{\mu \nu}(x) \hat{F}^{\lambda \rho}(x)\right] * e^{i k \cdot x} \\
T_{\nu}^{\mu}(k) & =\operatorname{Tr} \int d^{4} x L_{*}\left[W(x, C)\left(\hat{F}_{\alpha}^{\mu}(x) \hat{F}_{\nu}^{\alpha}(x)-\frac{1}{4} \delta_{\nu}^{\mu} \hat{F}^{\lambda \rho} \hat{F}_{\lambda \rho}\right)\right] * e^{i k \cdot x} \\
\Sigma_{\nu}^{\mu}(k) & =\operatorname{Tr} \int d^{4} x L_{*}\left[W(x, C)\left(\hat{F}_{\nu}^{\mu}+\hat{F}_{\alpha}^{\mu} \hat{F}_{\beta}^{\alpha} \hat{F}_{\nu}^{\beta}-\frac{1}{4} \hat{F}^{\lambda \rho} \hat{F}_{\lambda \rho} \hat{F}_{\nu}^{\mu}\right)\right] * e^{i k \cdot x}
\end{aligned}
$$

Recall that $L_{*}$ was defined in (1.2) and that in the non-Abelian case it also includes the matrix orderings. In the above definitions, the indices are raised and lowered by the open string metric. In (3.10), the superscripts $(i), i=1,2$ corresponds to taking trace in $U\left(N_{i}\right), i=1,2$ respectively and the subscript $\left.\right|_{4}$ in the last term of (3.10) means taking only the terms with four factors of the gauge field. The expansions of the operators (3.11)-(3.14) in $\hat{A}$ can be found using (2.14) and (2.16).

Equation (3.10) can be obtained from tree-level exchanges of the following momentum space action describing the couplings between the open and the closed string modes

$$
\begin{aligned}
S_{I}=\frac{\sqrt{2} \kappa_{10}}{\left(2 \pi G_{s}\right)} \int d^{4} k \sqrt{\operatorname{det} G}[ & \phi(-k) \mathcal{O}_{\phi}(k)+\chi(-k) \mathcal{O}_{\chi}(k) \\
& \left.+\frac{1}{2} h_{\mu}^{\nu}(-k) T_{\nu}^{\mu}(k)+\frac{1}{2} b_{\nu}^{\mu}(-k) \tilde{\Sigma}_{\mu}^{\nu}(k)\right]
\end{aligned}
$$

where $\phi, \chi, h_{\nu}^{\mu}, b_{\mu}^{\nu}$ are small fluctuations of the dilaton, axion, graviton (polarized along the brane directions) and NS-NS 2-form respectively. In (3.15) the closed string modes are canonically normalized and $\tilde{\Sigma}_{\mu}^{\nu}$ is given from $(3.14)$ by

$$
\tilde{\Sigma}_{\nu}^{\mu}(k)=\operatorname{Tr} \int d^{4} x L_{*}\left[W(x, C)\left(\left(\frac{1}{2 \kappa_{10}^{2}}\right)^{\frac{1}{4}} \hat{F}_{\nu}^{\mu}+\left(2 \kappa_{10}^{2}\right)^{\frac{1}{4}}\left(\hat{F}_{\alpha}^{\mu} \hat{F}_{\beta}^{\alpha} \hat{F}_{\nu}^{\beta}-\frac{1}{4} \hat{F}^{\lambda \rho} \hat{F}_{\lambda \rho} \hat{F}_{\nu}^{\mu}\right)\right)\right] * e^{i k \cdot x}
$$

Note while the indices of $T_{\nu}^{\mu}$ and $\Sigma_{\mu}^{\nu}$ are raised and lowered by the open string metric $G_{\mu \nu}$, those of $h_{\mu}^{\nu}$ and $b_{\nu}^{\mu}$ are raised and lowered by the closed string metric $g_{\mu \nu}$. So we should be careful that for instance the coupling $h_{\mu}^{\nu} T_{\nu}^{\mu}$ is different from $h_{\mu \nu} T^{\mu \nu}$. 
(3.15) gives the couplings of the lowest dimension gauge invariant operators in $\mathcal{N}=$ 4 noncommutative SYM to massless closed string modes in a flat space background. This identification should be helpful for understanding the supergravity description of the $\mathcal{N}=4$ noncommutative SYM theory $[22,23]$. We note that the operators $(3.11)-$ (3.14) may also have dependence on the scalar fields and fermions of the $\mathcal{N}=4$ theory. It would be interesting to work them out by supersymmetry or by looking at the amplitudes involving scalars and fermions.

\section{The Seiberg-Witten Map}

In this section we propose a closed form for the Seiberg-Witten map [6] between the ordinary and noncommutative Yang-Mills field. We shall only discuss the simpler $U(1)$ case. Our proposal is based on the earlier works on the Seiberg-Witten map [17, 28, $29,30,31,32,20]$ and the connection between open Wilson lines and $*_{n}$ operations discussed in section 2 . We shall first derive the map to first order in $\theta$, but to all orders in $\hat{A}$, using the results of $[28,29,31]$, and then try to generalize it to all orders in $\theta$. For definiteness we will restrict our discussion to four Euclidean dimensions, although our results in this and next sections apply invariably to any dimensions.

\subsection{The Seiberg-Witten Map to First Order in $\theta$}

Mathematically speaking, the Seiberg-Witten map can be understood as the transformation between star-products associated with cohomologically equivalent symplectic forms $[31,32]$. The first part of this subsection is a review of $[29,28,39,31]$. Consider two symplectic forms

$$
\omega_{\mu \nu}=\left(\theta^{-1}\right)_{\mu \nu}+F_{\mu \nu}, \quad B_{\mu \nu}=\left(\theta^{-1}\right)_{\mu \nu}
$$

where $\theta^{\mu \nu}$ is a constant anti-symmetric tensor and $F=d A$ is the field strength of a Abelian gauge field $A_{\mu}$. We shall assume that both $\omega$ and $B$ are non-degenerate. We can associate star products $*_{\omega}$ and $*_{B}$ with $\omega$ and $B$ respectively. $*_{B}$ is the standard Moyal product with a noncommutative parameter $\theta^{\mu \nu}$, while for general $F$, $*_{\omega}$ must be defined a la Kontsevich [40] and is much more complicated.

Since $B$ and $\omega$ differ by an exact form, it is possible to find a coordinate transformation $\lambda$ which maps $\omega$ to $B$, i.e. $\lambda: x \rightarrow y=y(x)$ so that

$$
\frac{\partial y^{\rho}}{\partial x^{\mu}} \frac{\partial y^{\lambda}}{\partial x^{\nu}} \omega_{\rho \lambda}(y)=B_{\mu \nu}(x)=\text { constant }
$$

Thus the symplectic structures defined by $\omega$ and $B$ belong to the same equivalence class and the two star products $*_{\omega}$ and $*_{B}$ must also be equivalent. More explicitly, 
there exists a map $\mathcal{D}$ acting on the space of functions which satisfies

$$
\mathcal{D}\left(f *_{\omega} g\right)=\mathcal{D} f *_{B} \mathcal{D} g
$$

In particular the noncommutative Yang-Mills field is defined by

$$
X^{\mu}(x)=\mathcal{D} y^{\mu} \equiv x^{\mu}+\theta^{\mu \nu} \hat{A}_{\nu} .
$$

It can be shown that an ordinary gauge transformation of $A$ induces a noncommutative gauge transformation of $\hat{A}$ and vice versa [31]. Heuristically, the physical information originally contained in $F$ is transferred to $\hat{A}$ through the map $\mathcal{D}$.

Although in principle $\mathcal{D}$ can be worked out in the formalism of Kontsevich [40] order by order in $\theta$ and $A$ (see e.g. $[31,32]$ ), its structure appears to be quite complicated. In this paper we pursue a slightly different route. We first find the map at first order in $\theta$ but to all orders in $\hat{A}$ and then try to generalize it to all orders in $\theta$.

To first order in $\theta, \mathcal{D}$ is given by $\mathcal{D}=\lambda^{*}[29,28,31]$, where $\lambda$ is the diffeomorphism (4.2) which maps $\omega$ to $B$. At this order, (4.3) reduces to an equivalence between the associated Poisson brackets

$$
\lambda^{*}\{f, g\}_{w}=\left\{\lambda^{*} f, \lambda^{*} g\right\}_{B},
$$

where $\lambda^{*} f=f \circ \lambda=f(y(x))$ and \{\}$_{\omega},\{\}_{B}$ are the Poisson Brackets with respect to $\omega$ and $B$ respectively. The counterpart of (4.4) is

$$
y^{\mu}(x)=\lambda^{*} y^{\mu}=x^{\mu}+\theta^{\mu \nu} a_{\nu}(x)
$$

where we have used a different symbol $a_{\mu}$ to denote the noncommuative gauge field $\hat{A}_{\mu}$ at the lowest order in $\theta$.

In (4.5) setting $f(y)=y^{\mu}, g(y)=y^{\nu}$, we obtain that

$$
\left(\omega^{-1}\right)^{\mu \nu}(y(x))=\left\{x^{\mu}+\theta^{\mu \sigma} a_{\sigma}(x), x^{\nu}+\theta^{\nu \rho} a_{\rho}(x)\right\}_{B}=-\left(\theta\left(f-\theta^{-1}\right) \theta\right)^{\mu \nu}
$$

with the corresponding field strength for $a$ given by

$$
f_{\mu \nu}=\partial_{\mu} a_{\nu}-\partial_{\nu} a_{\mu}+\theta^{\lambda \rho} \partial_{\lambda} a_{\mu} \partial_{\rho} a_{\nu}
$$

Equations (4.1) and (4.7) lead to

$$
F_{\mu \nu}(y(x))=\left(\frac{1}{1-f \theta} f\right)_{\mu \nu}(x) .
$$

Since

$$
F_{\mu \nu}(k)=\int d^{4} y F_{\mu \nu}(y) e^{i k \cdot y}
$$


we obtain

$$
F_{\mu \nu}(k)=\int d^{4} x \operatorname{det} M\left(\frac{1}{1-f \theta} f\right)_{\mu \nu} e^{i k_{\rho}\left(x^{\rho}+\theta^{\rho \sigma} a_{\sigma}\right)}
$$

where $M$ is the Jacobi matrix of the coordinate transformation from $y$ to $x$, i.e.

$$
M_{\mu}^{\nu}=\frac{\partial y^{\nu}}{\partial x_{\mu}}=\delta_{\mu}^{\nu}+\theta^{\nu \lambda} \partial_{\mu} a_{\lambda}
$$

Note that

$$
\theta^{\mu \nu} \frac{\partial y^{\lambda}}{\partial x_{\mu}} \frac{\partial y^{\rho}}{\partial x_{\nu}}=\left[\theta\left(\theta^{-1}-f\right) \theta\right]^{\lambda \rho} \Rightarrow \operatorname{det} M=\sqrt{\operatorname{det}(1-\theta f)}
$$

From (4.10) and (4.12) we finally get

$$
F_{\mu \nu}(k)=\int d^{4} x \sqrt{\operatorname{det}(1-\theta f)}\left(\frac{1}{1-f \theta} f\right)_{\mu \nu} e^{i k_{\rho}\left(x^{\rho}+\theta^{\rho \sigma} a_{\sigma}\right)} .
$$

In the above we have established an explicit transformation between an Abelian vector field $A$ with field strength $F$ and a "Poisson" vector field $a$ whose field strength $f$ (4.8) has a nonlinear term given by the Poisson bracket of $a$. It can be checked $[28,31$, 32 ] that an ordinary gauge transformation of $A$ is equivalent to a gauge transformation of $a$ of the form

$$
a_{\mu} \rightarrow a_{\mu}+\partial_{\mu} \lambda(x)-\left\{\lambda, a_{\mu}\right\}_{B}=a_{\mu}+\partial_{\mu} \lambda(x)-\theta^{\nu \rho} \partial_{\nu} \lambda \partial_{\rho} a_{\mu}
$$

Equations (4.8) and (4.14) are precisely the formulas for the field strength and gauge transformations of a noncommutative gauge field $\hat{A}$ to first order in $\theta$ (see e.g. equations (A.8)). Thus (4.13) gives us a closed form of the Seiberg-Witten map to first order in $\theta$.

\subsection{A Proposal for the Seiberg-Witten Map}

Now let us try to generalize (4.13) to all orders in $\theta$. First we replace $a$ in (4.13) by $\hat{A}$, and $f(4.8)$ by the noncommutative field strength $\hat{F}$,

$$
\hat{F}_{\mu \nu}=\partial_{\mu} \hat{A}_{\nu}-\partial_{\nu} \hat{A}_{\mu}-i \hat{A}_{\mu} * \hat{A}_{\nu}+i \hat{A}_{\nu} * \hat{A}_{\mu}
$$

from which procedure we get

$$
F_{\mu \nu}(k)=\int d^{4} x \sqrt{\operatorname{det}(1-\theta \hat{F})}\left(\frac{1}{1-\hat{F} \theta} \hat{F}\right)_{\mu \nu} e^{i k_{\rho}\left(x^{\rho}+\theta^{\rho \sigma} \hat{A}_{\sigma}\right)} .
$$


Note that since $x$ satisfies the commutation relations

$$
\left[x^{\mu}, x^{\nu}\right]=i \theta^{\mu \nu}
$$

the exponential factor in equation (4.16) is nothing but a straight open Wilson line [14], i.e.

$$
e^{i k_{\rho}\left(x^{\rho}+\theta^{\rho \sigma} \hat{A}_{\sigma}\right)}=W(x, C) * e^{i k \cdot x}
$$

where $C$ is given by $(2.3)$ and $W(x, C)$ by (2.2). Substituting (4.18) into (4.16) we get

$$
F_{\mu \nu}(k)=\int d^{4} x \sqrt{\operatorname{det}(1-\theta \hat{F})}\left(\frac{1}{1-\hat{F} \theta} \hat{F}\right)_{\mu \nu} W(x, C) e^{i k \cdot x}
$$

The manipulations above are somewhat formal since we have not specified an ordering or product structure for the fields inside the integral. While equation (4.19) reduces correctly to (4.13) to first order in $\theta$, to find the exact form of the map, we need to

1. specify an precise product structure and ordering for the integrand.

2. find any additional dependence on $\hat{F}$ or $\hat{A}$ which vanishes to first order in $\theta .^{9}$

In $[17,20]$, the Seiberg-Witten map was found to cubic order in $\hat{A}$ while exact in $\theta$. Their results are

$$
\begin{aligned}
F_{\mu \nu} & =\hat{F}_{\mu \nu}+\theta^{\lambda \rho}\left(\partial_{\rho}\left(\hat{A}_{\lambda} *^{\prime} \hat{F}_{\mu \nu}\right)+\frac{1}{2} \hat{F}_{\mu \nu} *^{\prime} \hat{F}_{\lambda \rho}-\hat{F}_{\mu \lambda} *^{\prime} \hat{F}_{\nu \rho}\right) \\
& +\frac{1}{2} \theta^{\lambda \rho} \theta^{\tau \sigma}\left(\partial_{\lambda} \partial_{\tau}\left[\hat{F}_{\mu \nu} \hat{A}_{\sigma} \hat{A}_{\rho}\right] *_{3}-\partial_{\tau}\left[\hat{F}_{\lambda \rho} \hat{F}_{\mu \nu} \hat{A}_{\sigma}\right] *_{3}+2 \partial_{\tau}\left[\hat{F}_{\mu \lambda} \hat{F}_{\nu \rho} \hat{A}_{\sigma}\right] *_{3}\right) \\
& -\theta^{\lambda \rho} \theta^{\tau \sigma}\left(\frac{1}{2}\left[\hat{F}_{\mu \lambda} \hat{F}_{\nu \rho} \hat{F}_{\tau \sigma}\right] *_{3}-\frac{1}{8}\left[\hat{F}_{\mu \nu} \hat{F}_{\lambda \rho} \hat{F}_{\tau \sigma}\right] *_{3}-\frac{1}{4}\left[\hat{F}_{\mu \nu} \hat{F}_{\rho \tau} \hat{F}_{\lambda \sigma}\right] *_{3}+\left[\hat{F}_{\lambda \tau} \hat{F}_{\mu \sigma} \hat{F}_{\nu \rho}\right] *_{3}\right) \\
& +O\left(\hat{A}^{4}\right) .
\end{aligned}
$$

From our results in section 2 it is easy to see that the gauge invariant completion of $(4.20)$ is

$$
\begin{aligned}
F_{\mu \nu}(k) & =\int d^{4} x e^{i k \cdot x} L_{*}\left[W ( x , C ) \left(\hat{F}_{\mu \nu}+\frac{1}{2} \theta^{\lambda \rho} \hat{F}_{\mu \nu} \hat{F}_{\lambda \rho}-\theta^{\lambda \rho} \hat{F}_{\mu \lambda} \hat{F}_{\nu \rho}\right.\right. \\
& \left.\left.-\theta^{\lambda \rho} \theta^{\tau \sigma}\left(\frac{1}{2} \hat{F}_{\mu \lambda} \hat{F}_{\nu \rho} \hat{F}_{\tau \sigma}-\frac{1}{8} \hat{F}_{\mu \nu} \hat{F}_{\lambda \rho} \hat{F}_{\tau \sigma}-\frac{1}{4} \hat{F}_{\mu \nu} \hat{F}_{\rho \tau} \hat{F}_{\lambda \sigma}+\hat{F}_{\lambda \tau} \hat{F}_{\mu \sigma} \hat{F}_{\nu \rho}\right)\right)\right],
\end{aligned}
$$

\footnotetext{
${ }^{9}$ More precisely, from (4.13), by first order in $\theta$, we mean taking $\theta \ll 1$ and $\theta \hat{A} \sim O(1)$.
} 
where $L_{*}$ was defined in (1.2). We further note that the $\hat{F}$ terms which multiply the open Wilson line in (4.21) are nothing but precisely the expansion of

$$
\sqrt{\operatorname{det}(1-\theta \hat{F})}\left(\frac{1}{1-\hat{F} \theta} \hat{F}\right)_{\mu \nu}
$$

to cubic order in $\hat{F}$.

Thus by comparing (4.19) to (4.21) it is tempting to conjecture that the exact Seiberg-Witten map is given by

$$
F_{\mu \nu}(k)=\int d^{4} x L_{*}\left[\sqrt{\operatorname{det}(1-\theta \hat{F})}\left(\frac{1}{1-\hat{F} \theta} \hat{F}\right)_{\mu \nu} W(x, C)\right] e^{i k \cdot x}
$$

where the (4.22) part of the integrand should be understood as a power series of $\hat{F}$. Equation (4.23) is gauge invariant by construction. It reduces to (4.13) when expanded to first order in $\theta$ and when expanded to the third order in $\hat{A}$ while keeping the exact $\theta$-dependence it recovers (4.20). We are currently unable to prove (4.23) to all orders in $\theta$. However (4.23) does appear to be the simplest possibility that fits all the available information. We shall see in the next section that (4.23) also gives rise to the correct formula when we ignore all the derivatives on $\hat{F}$.

One way to check that (4.23) is the right answer is to write it in a form

$$
F_{\mu \nu}(k)=-i k_{\mu} A_{\nu}+i k_{\nu} A_{\mu}
$$

Since in the $U(1)$ case, $F_{\mu \nu}$ is invariant under the noncommutative gauge transformations, $A_{\mu}$ found from (4.24) must transform by an ordinary gauge transformation under a noncommutative gauge transformation in $\hat{A}$.

Alternatively one may try to show that (4.23) satisfies the set of differential equations derived in [6] for $\hat{A}$ and $\hat{F}$ to ensure the gauge equivalence relations:

$$
\begin{aligned}
\delta \hat{A}_{a} & =-\frac{1}{4} \delta \theta^{k l}\left[\hat{A}_{k} *\left(\partial_{l} \hat{A}_{a}+\hat{F}_{l a}\right)+\left(\partial_{l} \hat{A}_{a}+\hat{F}_{l a}\right) * \hat{A}_{k}\right] \\
\delta \hat{F}_{a b} & =\frac{1}{4} \delta \theta^{k l}\left[2 \hat{F}_{a k} * \hat{F}_{b l}+2 \hat{F}_{b l} * \hat{F}_{a k}-\hat{A}_{k} *\left(\hat{D}_{l} \hat{F}_{a b}+\partial_{l} \hat{F}_{a b}\right)-\left(\hat{D}_{l} \hat{F}_{a b}+\partial_{l} \hat{F}_{a b}\right) * \hat{A}_{k}\right]
\end{aligned}
$$

However, the solutions of the equation (4.25) generally depend on the choice of integration paths. Solutions corresponding to different paths are related by gauge transformations or field redefinitions (see e.g. [41, 42]). Equivalently one may add terms which are gauge transformations and field redefinitions to the right hand sides of (4.25). That is, (4.25) is just one of many possible "Seiberg-Witten" equations. Thus it is not very clear 
what the precise prescription is for checking that (4.23) satisfies gauge equivalence. As an example, it is easy to check that (4.20) only satisfies (4.25) to quadratic order in $\hat{A}$ if we take the variation of $\theta$ to be proportional to itself, i.e. $\delta \theta^{i j} \propto \theta^{i j}{ }^{10}$ while at cubic order one may have to use additional gauge transformations and field redefinitions ${ }^{11}$.

Since $*_{n}$ is fully symmetric with respect to all its arguments, the prescription of (4.23) is reminiscent of the symmetrized trace prescription of the non-Abelian BornInfeld action proposed by Tseytlin [43]. The trace here is over the infinitely dimensional Hilbert space defined by (4.17). It would be interesting to investigate the possible connection through the equivalence between the Born-Infeld actions for the commutative and noncommutative gauge fields. We finally note that by using the definition of $L_{*}$ and $*_{n}$ in the general $U(N)$ case, it is not inconceivable that if (4.23) is correct, it may apply to the general $U(N)$ case.

\section{Equivalence of Born-Infeld Actions Revisited}

In this section we give a direct proof of the equivalence between the Born-Infeld actions for the ordinary and noncommutative gauge fields, discovered in [6]. We shall see that the explicit transformation (4.23) helps clarify certain aspects of the equivalence.

Since the Born-Infeld action describes the effective action of slowly varying fields on a D-brane, we can ignore terms involving derivatives of $\hat{F}$ in (4.23). This means we can replace the $*_{n}$ products by ordinary products. However we shall still keep the exponential factor of $\hat{A}$ since $\partial_{\nu} \hat{A}_{\mu}$ is of the same order as $\hat{F}$. Thus we now have

$$
F_{\mu \nu}(k)=\int d^{4} x \sqrt{\operatorname{det}(1-\theta \hat{F})}\left(\frac{1}{1-\hat{F} \theta} \hat{F}\right)_{\mu \nu} e^{i k_{\rho}\left(x^{\rho}+\theta^{\rho \sigma} \hat{A}_{\sigma}\right)}
$$

with ordinary products between various fields. Equation (5.1) is almost exactly the same as (4.13) except that here $\hat{F}$ has the full nonlinear structure (4.15). The steps from (4.9) to (4.13) suggests that we may derive an analogue of equation (4.9) for $\hat{F}$. Define $X$ as

$$
X^{\mu}(x)=x^{\mu}+\theta^{\mu \nu} \hat{A}_{\nu}(x)
$$

When $x$ satisfies the commutation relation (4.17), then

$$
\left[X^{\mu}, X^{\nu}\right]=i \Theta^{\mu \nu}=i((1-\theta \hat{F}) \theta)^{\mu \nu}
$$

\footnotetext{
${ }^{10}$ We note that to first order in $\theta$, it is indeed possible to check that (4.13) satisfies the Seiberg-Witten equation (4.25) if we choose the path of variation to be $\delta \theta^{i j} \propto \theta^{i j}$.

${ }^{11} \mathrm{I}$ would like to thank T. Mehen for correspondence about this point.
} 
In particular

$$
\sqrt{\operatorname{det} \Theta}=\sqrt{\operatorname{det} \theta} \sqrt{\operatorname{det}(1-\theta \hat{F})}
$$

and now (5.1) can be written as

$$
F_{\mu \nu}(k)=\int d^{4} x \frac{\sqrt{\operatorname{det} \Theta}}{\sqrt{\operatorname{det} \theta}}\left(\frac{1}{1-\hat{F} \theta} \hat{F}\right)_{\mu \nu} e^{i k \cdot X}
$$

Recall that the integration over $x$ can be understood as taking the trace over the Hilbert space defined by (4.17), i.e.

$$
\int d^{4} x \frac{1}{\sqrt{\operatorname{det} \theta}} \rightarrow \operatorname{Tr}
$$

Considering (5.3) and (5.6) and ignoring any derivatives on $\hat{F}$ we may rewrite (5.5) as

$$
F_{\mu \nu}(k)=\int d^{4} X\left(\frac{1}{1-\hat{F} \theta} \hat{F}\right)_{\mu \nu} e^{i k \cdot X}
$$

This rather looks like a Fourier transformation in the variable $X$ and thus we find

$$
F_{\mu \nu}(X(x))=\left(\frac{1}{1-\hat{F} \theta} \hat{F}\right)_{\mu \nu}(x)
$$

Equation (5.8) is our proposal for the Seiberg-Witten map in cases in which we can ignore the derivatives of $\hat{F}$ and $F$. In some sense (5.8) is very elegant in that it encodes the complicated relations between $F$ and $\hat{F}$ through a change of measure from $x$ to $X(x)=x+\theta \hat{A}$. In particular when $F=$ const it reduces to the familiar result in $[6]$.

In Appendix $\mathrm{C}$ we present an alternative derivation of equation (5.8) based on the equivalence relation (4.3) and the model of Catteneo-Felder [44]. That the two approaches give the same result (5.8) gives further support to our proposal (4.23).

It is now a simple matter to demonstrate the equivalence of the Born-Infeld actions using (5.8). Using the relations between the open and closed string moduli [6],

$$
\begin{aligned}
& \frac{1}{g+B}=\frac{1}{G+\Phi}+\frac{\theta}{2 \pi \alpha^{\prime}} \\
& \frac{1}{g_{s}} \sqrt{\operatorname{det}(g+B)}=\frac{1}{G_{s}} \sqrt{\operatorname{det}(G+\Phi)} .
\end{aligned}
$$


we have

$$
\begin{aligned}
S_{B I} & =\frac{1}{(2 \pi)^{3} \alpha^{\prime 2} g_{s}} \int d^{4} X \sqrt{\operatorname{det}\left(g+B+2 \pi \alpha^{\prime} F(X)\right)} \\
& =\frac{1}{(2 \pi)^{3} \alpha^{\prime 2} g_{s}} \sqrt{\operatorname{det}(g+B)} \int d^{4} x \sqrt{\operatorname{det}(1-\theta \hat{F})} \sqrt{\operatorname{det}\left(1+\frac{1}{g+B} 2 \pi \alpha^{\prime} \frac{1}{1-\hat{F} \theta} \hat{F}\right)} \\
& =\frac{1}{(2 \pi)^{3} \alpha^{\prime 2} G_{s}} \sqrt{\operatorname{det}(G+\Phi)} \\
& \times \int d^{4} x \sqrt{\operatorname{det}(1-\theta \hat{F})} \sqrt{\operatorname{det}\left(1+\left(\frac{1}{G+\Phi}+\frac{\theta}{2 \pi \alpha^{\prime}}\right) 2 \pi \alpha^{\prime} \hat{F} \frac{1}{1-\theta \hat{F}}\right)} \\
& =\frac{1}{(2 \pi)^{3} \alpha^{\prime 2} G_{s}} \int d^{4} x \sqrt{\operatorname{det}\left(G+\Phi+2 \pi \alpha^{\prime} \hat{F}\right)}
\end{aligned}
$$

In the second line above we have used equation (5.8) and made a coordinate change inside the integral. In the third line we substituted the relations (5.9).

In [6], the equivalence between the ordinary and noncommutative Born-Infeld Lagragians was proved by showing that the noncommutative BI Lagrangian is independent of the $\theta$ parameter up to total derivative terms. It was pointed out there that, for constant $F$, where the map is given by

$$
F_{\mu \nu}=\left(\frac{1}{1-\hat{F} \theta} \hat{F}\right)_{\mu \nu}
$$

the two Lagrangians are actually different. The reason is that for constant $F$ it is not legitimate to throw away total derivatives which may contribute through boundary terms ${ }^{12}$. What (5.10) says is that the information lost in the boundary terms is precisely encoded in the measure change between $X$ and $x$, and when included correctly, the two actions are indeed equivalent. Consider a simple example with $\hat{F}=\frac{1}{\theta}$ in a finite region of space and zero outside. From equation (5.11) the ordinary $F$ field is infinite in this limit and the two BI lagrangians are obviously different. However the measure change from $x$ to $X$ indicates the region in which $F$ is nonzero shrinks to zero size at the same time, ensuring that the whole actions are equivalent.

\section{Discussion}

In this paper we have discussed that the natural way to understand the appearance of the $*_{n}$ operations in various places of noncommutative gauge theories is through

\footnotetext{
${ }^{12}$ The discussion of this paragraph is developed with S. Minwalla.
} 
the open Wilson lines. These include the one-loop effective action of noncommutative gauge theories, the couplings between massless closed and open string modes, and the Seiberg-Witten map between the ordinary and noncommutative Yang-Mills fields. One common theme in the discussion is the need for gauge invariance and the Wilson line is the natural object to realize that. In all cases the $L_{*}$ prescription (1.2) - i.e. smearing operators along a straight open Wilson line-played an important role. In one-loop amplitudes and closed-open string couplings the $L_{*}$ prescription can be understood from the "stretched string effect" discussed in [25, 15] and has its origin in the integrations over the vertex operator insertions on the worldsheet. It should be interesting to understand the physical reason for its appearance in the Seiberg-Witten map. Of course, the above cases are not unrelated to each other, e.g. one-loop open string amplitudes are related to the closed-open tree-level amplitudes by factorization. Also since closed string modes have a simple off-shell coupling to ordinary Yang-Mills field variables (e.g. through ordinary Born-Infeld action), it is not hard to imagine that the presence of open Wilson lines in the closed-noncommutative Yang-Mills field couplings and the Seiberg-Witten map may be of the same origin.

The explicit couplings worked out in section 3 between the modes of noncommutative gauge theory and massless closed strings in flat space should be useful for understanding the operator-field correspondence in the supergravity description of the theory. Based on the examples found in this paper, we may try to speculate what is the general pattern for the operator-field matching. A most naive expectation would be that we start with operator-field matching in the ordinary AdS/CFT correspondence, replace the Yang-Mills field variables by their noncommutative counterparts and attach the resulting operators to a straight Wilson line with $L_{*}$ ordering to obtain the field-operator matching in the noncommutative case. It should be interesting to work out more examples by using supersymmetry or by considering amplitudes involving the scalar fields and fermions. However we caution that the situation might be much more complicated since in the noncommutative case the supergravity background [22, 23] has rather complicated mixings between small fluctuations due to non-trivial background fields. This might imply that the operator-field matching in the supergravity description is more intricate than the couplings we observed between the noncommutative SYM and closed string modes in flat space.

The closed form of the Seiberg-Witten map and its cousin in the slowly-varying field case can also have various applications. For example it should be useful for studying questions like the behaviors of solitons in the presence of a $B$-field (e.g. $[6,45,46]$ ) and constraining the structure of higher-derivative terms in the Born-Infeld action (e.g. [47, 39]). From the string theory point of view, the map follows from that ordinary and noncommutative field variables arise from different regularizations of the same 
string worldsheet theory and their respective effective actions correspond to different off-shell extensions of the on-shell string amplitudes. An explicit form of the map in the field theory context might be helpful for studying the more difficult question of finding the relations between different off-shell extensions in string theory, a question which is of much importance in string field theory.

\section{Acknowledgments}

I have benefited from useful discussions and correspondences with T. Banks, M. Douglas, R. Gopakumar, D. Gross, C. Hofman, G. Moore, M. Mariño, T. Mehen, J. Michelson, S. Minwalla, B. Pioline, S.-J. Rey, M. Rozali and P. Schupp. I thank J. Michelson for encouragement and for a critical reading of the manuscript. The author also thanks the Harvard group, where a portion of this work was performed, for hospitality and fruitful discussion. This work was supported by DOE grant \#DE-FG02-96ER40559.

\section{Appendix}

\section{A. Notations and Conventions}

Here we list our conventions about noncommutative gauge theory. We will restrict our discussion to a noncommutative $\mathbb{R}^{4}$, in which

$$
\left[x^{\mu}, x^{\nu}\right]=i \theta^{\mu \nu}
$$

where c-number $\theta$ is antisymmetric and non-degenerate. The algebra of functions on this space is given by the $*$-product:

$$
f(x) * g(x)=\left.\exp \left[\frac{i}{2} \theta^{\mu \nu} \frac{\partial}{\partial x^{\mu}} \frac{\partial}{\partial y^{\nu}}\right] f(x) g(y)\right|_{x=y} .
$$

In momentum space,

$$
f\left(k_{1}\right) * g\left(k_{2}\right)=f\left(k_{1}\right) g\left(k_{2}\right) \exp \left(-\frac{i}{2} k_{1} \times k_{2}\right)
$$

where

$$
k_{1} \times k_{2} \equiv k_{1 \mu} \theta^{\mu \nu} k_{2 \nu}
$$

and the right hand side of the equation (A.3) is given by the ordinary product. 
The action for a noncommutative gauge theory is

$$
S=-\frac{1}{4 g^{2}} \int d^{4} x \operatorname{Tr} \hat{F}_{\mu \nu} * \hat{F}^{\mu \nu}
$$

where the noncommutative gauge field strength is

$$
\hat{F}_{\mu \nu}=\partial_{\mu} \hat{A}_{\nu}-\partial_{\nu} \hat{A}_{\mu}-i \hat{A}_{\mu} * \hat{A}_{\nu}+i \hat{A}_{\nu} * \hat{A}_{\mu}
$$

Under an infinitesimal gauge transformation,

$$
\begin{aligned}
\delta_{\hat{\lambda}} \hat{A}_{\mu} & =\partial_{\mu} \hat{\lambda}+i \hat{\lambda} * \hat{A}_{\mu}-i \hat{A}_{\mu} * \hat{\lambda} \\
\delta_{\hat{\lambda}} \hat{F}_{\mu \nu} & =i \hat{\lambda} * \hat{F}_{\mu \nu}-i \hat{F}_{\mu \nu} * \hat{\lambda}
\end{aligned}
$$

To first order in $\theta$ the above formulas for field strength and gauge transformations become

$$
\begin{aligned}
\hat{F}_{\mu \nu} & =\partial_{\mu} \hat{A}_{\nu}-\partial_{\nu} \hat{A}_{\mu}+\theta^{\lambda \rho} \partial_{\lambda} \hat{A}_{\mu} \partial_{\rho} \hat{A}_{\nu}=\partial_{\mu} \hat{A}_{\nu}-\partial_{\nu} \hat{A}_{\mu}+\left\{\hat{A}_{\mu}, \hat{A}_{\nu}\right\}_{\theta^{-1}} \\
\delta_{\hat{\lambda}} \hat{A}_{\mu} & =\partial_{\mu} \hat{\lambda}-\theta^{\rho \sigma} \partial_{\rho} \hat{\lambda} \partial_{\sigma} \hat{A}_{\mu}=\partial_{\mu} \hat{\lambda}-\left\{\hat{\lambda}, \hat{A}_{\mu}\right\}_{\theta^{-1}} \\
\delta_{\hat{\lambda}} \hat{F}_{\mu \nu} & =-\theta^{\rho \sigma} \partial_{\rho} \hat{\lambda} \partial_{\sigma} \hat{F}_{\mu \nu}=-\left\{\hat{\lambda}, \hat{F}_{\mu \nu}\right\}_{\theta^{-1}}
\end{aligned}
$$

where \{\}$_{\theta^{-1}}$ denotes the Poisson bracket with respect to the symplectic form $\left(\theta^{-1}\right)_{\mu \nu}$.

A Wilson line is given by

$$
\begin{aligned}
& W(x, C) \\
& =P_{*} \exp \left(i \int_{0}^{1} d \sigma \partial_{\sigma} \xi^{\mu}(\sigma) \hat{A}_{\mu}(x+\xi(\sigma))\right) \\
& =\lim _{\Delta x_{j} \rightarrow 0} \prod_{j}\left[1+i \hat{A}\left(x_{j}\right) \cdot \Delta x_{j}\right]_{*} \\
& =\sum_{n=0}^{\infty} i^{n} \int_{0}^{1} d \sigma_{1} \int_{\sigma_{1}}^{1} d \sigma_{2} \cdots \int_{\sigma_{n-1}}^{1} d \sigma_{n} \partial_{\sigma_{1}} \xi^{\mu_{1}} \cdots \partial_{\sigma_{n}} \xi^{\mu_{n}} \hat{A}_{\mu_{1}}\left(x+\xi\left(\sigma_{1}\right)\right) * \cdots * \hat{A}_{\mu_{n}}\left(x+\xi\left(\sigma_{n}\right)\right)
\end{aligned}
$$

\section{B. The $*_{n}$ Operations}

$*_{n}$ is an $n$-ary operation defined on the space of $n$-functions [15]. Its introduction was motivated from the structure of the one-loop non-planar amplitudes of the noncommutative gauge theories; for more details see $[15,21]$. 
It is convenient to define the $*_{n}$ operation in the momentum space, i.e.

$$
*_{n}\left[f_{1}(x), \cdots, f_{n}(x)\right]=\prod_{i=1}^{n}\left(\int \frac{d^{4} k_{i}}{(2 \pi)^{4}}\right) e^{i\left(k_{1}+\cdots+k_{n}\right) \cdot x} *_{n}\left[f_{1}\left(k_{1}\right), f_{2}\left(k_{2}\right), \cdots, f_{n}\left(k_{n}\right)\right] \text {. }
$$

In the $U(1)$ case, we define:

$$
*_{n}\left[f_{1}\left(k_{1}\right), f_{2}\left(k_{2}\right), \cdots, f_{n}\left(k_{n}\right)\right]=f_{1}\left(k_{1}\right) f_{2}\left(k_{2}\right) \cdots f_{n}\left(k_{n}\right) J_{n}\left(k_{1}, \cdots, k_{n}\right)
$$

where the right hand side of the equation is given by the ordinary product and $J_{n}$ is $\left(\tau_{i j}=\tau_{i}-\tau_{j}\right)$ :

$$
J_{n}\left(k_{1}, \cdots, k_{n}\right)=\int_{0}^{1} d \tau_{1} \cdots \int_{0}^{1} d \tau_{n} \exp \left[-\frac{i}{2} \sum_{i<j}^{n}\left(k_{i} \times k_{j}\right)\left(2 \tau_{i j}-\epsilon\left(\tau_{i j}\right)\right)\right]
$$

with $k_{1} \times k_{2} \equiv k_{1 \mu} \theta^{\mu \nu} k_{2 \nu}$. In (B.3), the integrations over $\tau$ have their origin, in string theory amplitudes, as integrations over the vertex operator insertions along the worldsheet boundary. The integrand of (B.3) comes from the $\theta$-dependent part of the annulus propagators. To compare with the Moyal product, we note that in the momentum space the Moyal product of $n$ functions is given by

$$
f_{1}\left(k_{1}\right) * f_{2}\left(k_{2}\right) * \cdots * f_{n}\left(k_{n}\right)=f_{1}\left(k_{1}\right) f_{2}\left(k_{2}\right) \cdots f_{n}\left(k_{n}\right) \exp \left[-\frac{i}{2} \sum_{i<j}^{n}\left(k_{i} \times k_{j}\right)\right]
$$

In the general $U(N)$ case, where $f_{i}$ are $N \times N$-valued matrices, let $f_{i}=\sum_{a_{i}} f_{i}^{a_{i}} T^{a_{i}}$ where $T^{a_{i}}$ are a set of basis. Then

$$
*_{n}\left[f_{1}\left(k_{1}\right), f_{2}\left(k_{2}\right), \cdots, f_{n}\left(k_{n}\right)\right]=f_{1}^{a_{1}}\left(k_{1}\right) f_{2}^{a_{2}}\left(k_{2}\right) \cdots f_{n}^{a_{n}}\left(k_{n}\right) J_{n}\left(a_{1}, k_{1} ; \cdots ; a_{n}, k_{n}\right)
$$

with

$$
\begin{aligned}
& J_{n}\left(a_{1}, k_{1} ; \cdots ; a_{n}, k_{n}\right) \\
& =\left(\prod_{i=1}^{n} \int_{0}^{1} d \tau_{i}\right) P_{\tau}\left(T^{a_{1}} \cdots T^{a_{n}}\right) \exp \left[-\frac{i}{2} \sum_{i<j}^{n}\left(k_{i} \times k_{j}\right)\left(2 \tau_{i j}-\epsilon\left(\tau_{i j}\right)\right)\right]
\end{aligned}
$$

where $P_{\tau}$ denotes an ordering of matrices $T^{a_{i}}$ according to the ordering of $\tau_{i}$ and is motivated from the structure of the string amplitudes with Chan-Paton factors. 
Here we list the explicit expressions for $n=2,3$ in the $U(1)$ case which were found in $[17,25]$. When $n=2$,

$$
J_{2}=\frac{\sin \frac{k_{1} \times k_{2}}{2}}{\frac{k_{1} \times k_{2}}{2}}, \quad *_{2}(f(x), g(x)) \equiv f(x) \frac{\sin \left(\frac{1}{2} \theta^{\mu \nu} \overleftarrow{\partial_{\mu}} \overrightarrow{\partial_{\nu}}\right)}{\frac{1}{2} \theta^{\mu \nu} \overleftarrow{\partial_{\mu}} \overrightarrow{\partial_{\nu}}} g(x)
$$

When $n=3$,

$$
J_{3}\left(k_{1}, k_{2}, k_{3}\right)=\frac{\sin \left(\frac{k_{2} \times k_{3}}{2}\right) \sin \left(\frac{k_{1} \times\left(k_{2}+k_{3}\right)}{2}\right)}{\frac{\left(k_{1}+k_{2}\right) \times k_{3}}{2} \frac{k_{1} \times\left(k_{2}+k_{3}\right)}{2}}+\frac{\sin \left(\frac{k_{1} \times k_{3}}{2}\right) \sin \left(\frac{k_{2} \times\left(k_{1}+k_{3}\right)}{2}\right)}{\frac{\left(k_{1}+k_{2}\right) \times k_{3}}{2} \frac{k_{2} \times\left(k_{1}+k_{3}\right)}{2}} .
$$

and the corresponding $*_{3}$ is

$$
\begin{aligned}
& *_{3}[f(x), g(x), h(x)] \\
& \left.\equiv\left[\frac{\sin \left(\frac{\partial_{2} \times \partial_{3}}{2}\right) \sin \left(\frac{\partial_{1} \times\left(\partial_{2}+\partial_{3}\right)}{2}\right)}{\frac{\left(\partial_{1}+\partial_{2}\right) \times \partial_{3}}{2} \frac{\partial_{1} \times\left(\partial_{2}+\partial_{3}\right)}{2}}+\frac{\sin \left(\frac{\partial_{1} \times \partial_{3}}{2}\right) \sin \left(\frac{\partial_{2} \times\left(\partial_{1}+\partial_{3}\right)}{2}\right)}{\frac{\left(\partial_{1}+\partial_{2}\right) \times \partial_{3}}{2} \frac{\partial_{2} \times\left(\partial_{1}+\partial_{3}\right)}{2}}\right] f\left(x_{1}\right) g\left(x_{2}\right) h\left(x_{3}\right)\right|_{x_{i}=x}
\end{aligned}
$$

\section{An Alternative Derivation of the Seiberg-Witten Map in the Slowly-varying Field Case}

Here we give a different derivation of (5.8) of section 5 based on the equivalence relation (4.3) and the path integral representation of Kontsevich's star product derived in [44]. The derivation is very similar to that used in section 4.1.

It was shown in [44] that when a symplectic form $\omega$ is non-singular, the Kontsevich's star-product can be obtained from the following path integral ${ }^{13}$,

$$
f(y) *_{\omega} g(y)=\int_{Y(\infty)=y} D Y f(Y(1)) g(Y(0)) e^{i S}
$$

where $Y$ defines a two-dimensional field theory on a disk and $0,1, \infty$ are three distinct ordered points on the boundary of the disk. In (C.1) the action is given by

$$
S=\frac{1}{2} \int d^{2} \sigma \epsilon^{a b} \omega_{\mu \nu} \partial_{a} Y^{\mu} \partial_{b} Y^{\nu}
$$

In our case, from (4.1),

$$
\omega=\left(\theta^{-1}\right)_{\mu \nu}+F_{\mu \nu}
$$

\footnotetext{
${ }^{13}$ What we consider below is a special simplified case of more general discussions in [44].
} 
is an exact form and the action (C.2) reduces to an action defined on the boundary of the disk,

$$
S=\frac{1}{2} \int d \sigma\left[\left(\theta^{-1}\right)_{\mu \nu} Y^{\mu} \partial_{\sigma} Y^{\nu}+A_{\mu}(Y(\sigma)) \partial_{\sigma} Y^{\nu}\right]
$$

where $A_{\mu}$ is the gauge field for $F$ and (C.3) can be considered as the action of a string world-sheet action in the presence of a gauge field background in the Seiberg-Witten decoupling limit.

Let $Y^{\mu}(\sigma)=y^{\mu}+\xi^{\mu}(\sigma)$. When we can ignore the derivatives on $F$, the action can be simplified to yield

$$
S=\frac{1}{2} \int d \sigma\left[\theta_{\mu \nu}^{-1}+F_{\mu \nu}(y)\right] \xi^{\mu} \partial_{\sigma} \xi^{\nu}+O(\partial F)
$$

In this limit the structure of the star-product is also considerably simplified. Let us take $f=y^{\mu}$ and $g=y^{\nu}$ in (C.1), which leads to

$$
y^{\mu} *_{\omega} y^{\nu}=\frac{i}{2}\left(\omega^{-1}\right)^{\mu \nu}
$$

With equation (4.3) this implies

$$
\mathcal{D}\left(\left[y^{\mu}, y^{\nu}\right]_{*_{\omega}}\right)=\left[\mathcal{D} y^{\mu}, \mathcal{D} y^{\nu}\right]_{*_{B}}
$$

Now using (4.4), we find that

$$
i\left(\omega^{-1}\right)_{\mu \nu}(X(x))=\left[X^{\mu}, X^{\nu}\right]_{*}
$$

and

$$
F_{\mu \nu}(X(x))=\left(\frac{1}{1-\hat{F} \theta} \hat{F}\right)_{\mu \nu}(x)
$$

\section{References}

[1] A. Connes, M. R. Douglas and A. Schwarz, Noncommutative geometry and matrix theory: Compactification on tori, J. High Energy Phys. 02 (1998) 003; [hep-th/9711162].

[2] M. R. Douglas and C. Hull, D-branes and the Noncommutative Torus, J. High Energy Phys. 02 (1998) 008; [hep-th/9711165].

[3] F. Ardalan, H. Arfaei and M.M. Sheikh-Jabbari, Noncommutative Geometry from Strings and Branes, J. High Energy Phys. 02 (1999) 016; [hep-th/9810072]. 
[4] C.-S. Chu and P.-M. Ho, Noncommutative Open String And D-Brane, Nucl. Phys. B 550 (1999) 151-168, [hep-th/9812219]

[5] V. Schomerus, D-Branes and Deformation Quantization, J. High Energy Phys. 06 (1999) 030; [hep-th/9903205].

[6] N. Seiberg and E. Witten, String theory and noncommutative geometry, J. High Energy Phys. 09 (1999) 032; [hep-th/9908142].

[7] D. J. Gross and N. A. Nekrasov, Dynamics of Strings in Noncommutative Gauge Theory, PUPT-1945, ITEP-TH-39/00, NSF-ITP-00-71, hep-th/0007204.

[8] S. R. Das and S.-J. Rey, Open Wilson Lines in Noncommutative Gauge Theory and Tomography of Holographic Dual Supergravity, SNUST-000702, TIFR-TH/00-40, hep-th/0008042.

[9] A. Rajaraman and M. Rozali, Noncommutative Gauge Theory, Divergences and Closed Strings, J. High Energy Phys. 04 (2000) 033; [hep-th/0003227].

[10] D. J. Gross and N. A. Nekrasov, Solitons in Noncommutative Gauge Theory, hep-th/0010090.

[11] N. Ishibashi, S. Iso, H. Kawai, Y. Kitazawa, Wilson Loops in Noncommutative Yang Mills, KEK-TH-649, KUNS-1608, hep-th/9910004.

[12] J. Ambjorn, Y. M. Makeenko, J. Nishimura and R.J. Szabo, Finite N Matrix Models of Noncommutative Gauge Theory, J. High Energy Phys. 11 (1999) 029; [hep-th/9911041]; J. Ambjorn, Y. M. Makeenko, J. Nishimura and R.J. Szabo, Lattice Gauge Fields and Discrete Noncommutative Yang-Mills Theory, J. High Energy Phys. 05 (2000) 023; [hep-th/0004147].

[13] D. J. Gross, A. Hashimoto and N. Itzhaki, Observables of Non-Commutative Gauge Theories, NSF-ITP-00-94, hep-th/0008075.

[14] A. Dhar, and S. R. Wadia, A Note on Gauge Invariant Operators in Noncommutative Gauge Theories and the Matrix Model, hep-th/0008144.

[15] H. Liu and J. Michelson, *-TREK: The One-Loop N=4 Noncommutative SYM Action, hep-th/0008205.

[16] D. Zanon, Noncommutative perturbation in superspace, hep-th/0009196;

A. Santambrogio and D. Zanon, One-loop four-point function in noncommutative $\mathcal{N}=4$ Yang-Mills theory, hep-th/0010275.

[17] M. R. Garousi, Non-commutative world-volume interactions on D-brane and Dirac-BornInfled action, IPM/P-99/051, hep-th/9909214. 
[18] F. Ardalan and N.Sadooghi, Anomaly and Nonplanar Diagrams in Noncommutative Gauge Theories, hep-th/0009233.

[19] S. Hyun, Y. Kiem, S. Lee and C.-Y. Lee, Closed Strings Interacting with Noncommutative D-branes, Nucl. Phys. B 569 (2000) 262, [hep-th/9909059].

[20] T. Mehen and M. B. Wise, Generalized $\star$-Products, Wilson Lines and the Solution of the Seiberg-Witten Equations, hep-th/0010204.

[21] H. Liu and J. Michelson, to appear.

[22] A. Hashimoto and N. Itzhaki, Non-Commutative Yang-Mills and the AdS/CFT Correspondence, Phys. Lett. B 465 (1999) 142-147; [hep-th/9907166].

[23] J. M. Maldacena and J. G. Russo, Large N Limit of Non-Commutative Gauge Theories, J. High Energy Phys. 09 (1999) 025; [hep-th/9908134].

[24] D. Bigatti and L. Susskind, Magnetic Fields, Branes and Noncommutative Geometry, SUITP 99/39, KUL-TF 99/30, hep-th/9908056.

[25] H. Liu and J. Michelson, Stretched Strings in Noncommutative Field Theory, Phys. Rev. D 62 (2000) 066003, [hep-th/0004013].

[26] M. M. Sheikh-Jabbari, Open Strings in a B-field Background as Electric Dipoles, Phys. Lett. B 455 (1999) 129-134; [hep-th/9901080].

[27] Z. Yin, A Note on Space Noncommutativity, Phys. Lett. B 466 (1999) 234-238; [hep-th/9908152].

[28] L. Cornalba, D-brane Physics and Noncommutative Yang-Mills Theory, hep-th/9909081.

[29] N. Ishibashi, A Relation between Commutative and Noncommutative Descriptions of Dbranes, hep-th/9909176.

[30] K. Okuyama, A Path Integral Representation of the Map between Commutative and Noncommutative Gauge Fields, J. High Energy Phys. 03 (2000) 016; [hep-th/9910138].

[31] B. Jurco, P. Schupp, Noncommutative Yang-Mills from equivalence of star products, Eur.Phys.J. C14 (2000) 367-370; [hep-th/0001032].

[32] B. Jurco, P. Schupp, J. Wess, Noncommutative gauge theory for Poisson manifolds, Nucl. Phys. B 584 (2000) 784-794; [hep-th/0005005].

[33] J. M. Maldacena, Wilson loops in large N field theories, Phys. Rev. Lett. 80 (1998) 4859; [hep-th/9803002]. 
[34] S.-J. Rey and J. Yee, Macroscopic Strings as Heavy Quarks of Large N Gauge Theory and Anti-de Sitter Supergravity, hep-th/9803001.

[35] M. R. Douglas and W. Taylor, Branes in the Bulk of Anti-de Sitter Space, PUPT-1806, RU-98-32, hep-th/9807225.

[36] S. R. Das, Brane Waves, Yang-Mills Theories and Causality, J. High Energy Phys. 02 (1999) 012; [hep-th/9901004]

S. R. Das, Holograms of Branes in the Bulk and Acceleration Terms in SYM Effective Action, J. High Energy Phys. 06 (1999) 029, [hep-th/9905037].

[37] D. Bak and S.-J. Rey, Holographic View of Causality and Locality via Branes in AdS/CFT Correspondence, Nucl. Phys. B 572 (2000) 151; [hep-th/9902101].

[38] M. R. Douglas, D. Kabat, P. Pouliot and S. H. Shenker, D-branes and Short Distances in String Theory, Nucl. Phys. B 485 (1997) 85; [hep-th/9608024].

[39] L. Cornalba, Corrections to the Abelian Born-Infeld Action Arising from Noncommutative Geometry, J. High Energy Phys. 09 (2000) 017; [hep-th/9912293];

L. Cornalba, On the General Structure of the Non-Abelian Born-Infeld Action, hep-th/0006018.

[40] M. Kontsevich, Deformation Quantization of Poisson Manifolds, I, q-alg/9709040.

[41] T. Asakawa and I. Kishimoto, Comments on Gauge Equivalence in Noncommutative Geometry, J. High Energy Phys. 11 (1999) 024; [hep-th/9909139].

[42] S. Terashima, The Non-Abelian Born-Infeld Action and Noncommutative gauge theory, hep-th/0006058.

[43] A.A. Tseytlin, On non-abelian generalisation of Born-Infeld action in string theory, Nucl. Phys. B 501 (1997) 41; [hep-th/9701125].

[44] A. S. Cattaneo and G. Felder, A path integral approach to the Kontsevich quantization formula, Comm. Math. Phys. 212 (2000) 591, math.QA[9902090].

[45] M. Marino, R. Minasian, G. Moore and A. Strominger, Nonlinear Instantons from Supersymmetric p-Branes, J. High Energy Phys. 01 (2000) 005; [hep-th/9911206].

[46] S. Terashima, Instantons in the U(1) Born-Infeld Theory and Noncommutative Gauge Theory, Phys. Lett. B 477 (2000) 292; [hep-th/9911245].

[47] Y. Okawa, Derivative corrections to Dirac-Born-Infeld Lagrangian and non-commutative gauge theory, Nucl. Phys. B 566 (2000) 348; [hep-th/9909132];

Y. Okawa and S. Terashima, Constraints on effective Lagrangian of D-branes from noncommutative gauge theory, Nucl. Phys. B 584 (2000) 329; [hep-th/0002194]. 